\title{
Electronic structure of and quantum size effect in III-V and II-VI semiconducting nanocrystals using a realistic tight binding approach
}

\author{
Ranjani Viswanatha, ${ }^{1}$ Sameer Sapra, ${ }^{1}$ Tanusri Saha-Dasgupta, ${ }^{2}$ and D. D. Sarma ${ }^{1, *}$ \\ ${ }^{1}$ Solid State and Structural Chemistry Unit, Indian Institute of Science, Bangalore-560012, India \\ ${ }^{2}$ S.N. Bose Centre, Kolkatta, India
}

\begin{abstract}
We analyze the electronic structure of group III-V semiconductors obtained within full potential linearized augmented plane wave (FP-LAPW) method and arrive at a realistic and minimal tight-binding model, parametrized to provide an accurate description of both valence and conduction bands. It is shown that the cation $s p^{3}$ anion $s p^{3} d^{5}$ basis along with the next nearest neighbor model for hopping interactions is sufficient to describe the electronic structure of these systems over a wide energy range, obviating the use of any fictitious $s^{*}$ orbital, employed previously. Similar analyses were also performed for the II-VI semiconductors, using the more accurate FP-LAPW method compared to previous approaches, in order to enhance reliability of the parameter values. Using these parameters, we calculate the electronic structure of III-V and II-VI nanocrystals in real space with sizes ranging up to about $7 \mathrm{~nm}$ in diameter, establishing a quantitatively accurate description of the bandgap variation with sizes for the various nanocrystals by comparing with available experimental results from the literature.
\end{abstract}

\section{INTRODUCTION}

Semiconductor nanocrystals, with the tunability of their electronic and optical properties by the three-dimensional confinement of carriers, have attracted considerable interest as technologically important materials. ${ }^{1}$ Hence, the study of the quantum confinement in these semiconductors has been a subject of intense study. Though the first approach to obtain a quantitative understanding of the quantum confinement effects on the bandgap of the nanocrystal as a function of size was given by the effective mass approximation ${ }^{2}$ (EMA), it is well known to overestimate the bandgap in the lower size regime. In the past few decades, the theoretical predictability of the variation of bandgap as a function of size has increased due to the development of a host of different theoretical approaches, starting from the $a b$ initio methods ${ }^{3}$ to the semiempirical pseudopotential ${ }^{4,5}$ and tight binding ${ }^{6-12}$ (TB) approaches. Recently, the TB method has gained certain popularity, both because of its realistic description of structural and dielectric properties in terms of chemical bonds and its simplicity, enabling one to handle very large systems. The Slater-Koster suggestion of treating the TB model as an interpolation scheme ${ }^{13}$ has been widely used in various semiconductors. However, the intuitively appealing, nearest neighbor $s p^{3}$ model fails to explain even the indirect gap in most of the III-V semiconductors satisfactorily, especially at the $\mathrm{X}$ point. In order to mimic the influence of the excited $d$ states, Vogl et al. used the $s^{*}$ orbital, in an ad hoc manner. ${ }^{14}$ Though it could explain the bandgap at the X point correctly, the band curvatures were not properly described. Following the recognition of the importance of the $d$ states by the pseudopotential methods, Jancu et al. have recently performed a TB calculation using a $s p^{3} d^{5} s^{*}$ basis for both cations and anions and the nearest neighbor interactions on III-V as well as group IV semiconductors. ${ }^{7}$ The band dispersions obtained by this calculation is found to overcome most of the deficiencies of the earlier TB models, though the use of the $s^{*}$ orbital, originally included to account for the absence of the excited $d$ states, becomes more questionable with the inclusion of the $d$ orbitals in the basis. Moreover, the transferability of the TB parameters obtained from bulk $a b$ initio band structures to the nanometric regime remains a controversial issue.

With the advance of experimental techniques such as photoemission and inverse photoemission techniques, it is possible to map out the density of states (DOS) of both valence and conduction bands for the bulk materials as well as the nanocrystals. With the introduction of site and angularmomentum specific $\mathrm{x}$-ray emission and absorption techniques, it is also possible to study the partial density of states (PDOS). Hence, the need for a physically sound, minimal model without any fictitious orbital and supported by accurate parametrization is required to be able to provide realistic descriptions of both valence and conduction bands in contrast to simulating only the bandgap of these semiconductors. Our attempts 9,12 in this direction on the II-VI semiconductors suggest that much of the difficulties arise from inaccurate parametrization of the bulk band structures. In order to overcome these difficulties, we carried out a detailed analysis of the bulk band structure obtained within the highly accurate FP-LAPW method, supported by the recently developed new generation muffin-tin orbital (NMTO) $\operatorname{method}^{15}$ to obtain a physical, realistic, and minimal model with accurate parametrizations. Such a method not only obviates the need for the fictitious $s^{*}$ orbital, but has also been found to explain quantum confinement effects quite well in the II-VI semiconductors. ${ }^{10-12}$

Though the synthesis of high quality nanometric sized II-VI semiconductors is already very well established, the synthesis and studies of high quality III-V semiconducting nanocrystals are being increasingly reported in the recent literature. ${ }^{16-20}$ III-V semiconductors provide a material basis 
for a number of already existing commercial products, as well as new cutting edge electronic and optoelectronic devices, like heterostructure bipolar transistors, diode lasers, light emitting diodes, electro-optic modulators ${ }^{21}$ and in biology, as fluorescent labels. ${ }^{22}$ Hence, it becomes necessary to have an electronic structure model with accurate predictive abilities to describe the quantum confinement effects in these nanocrystals.

In order to achieve this, we study the band dispersions as well as the DOS and PDOS obtained from the $a b$ initio full potential linearized augmented plane-wave (FP-LAPW) method $^{23}$ to establish the relative importance of various orbital degrees of freedom involved in describing the valence as well as some of the low-lying conduction bands in various III-V semiconductors. In order to identify the dominant hopping interactions, we employ the muffin-tin orbital (MTO)based NMTO technique, ${ }^{15}$ which provides a unique scheme to derive a first-principles TB Hamiltonian, starting from the full local density approximation (LDA) calculation. The usefulness of this method has been demonstrated in a number of cases. ${ }^{24}$ With inputs from these $a b$ initio methods, we construct a minimal TB model and carry out a least-squarederror minimization procedure to fit the $\mathrm{TB}$ dispersions to the $a b$ initio ones, thereby defining the values of the TB parameters. Using the TB parameters thus obtained, we carry out a real space calculation using Lanczos algorithm for different sizes of the nanocrystals to obtain the dependence of the electronic structure on the size of the nanocrystals. We compare these calculated results with the experimentally determined bandgaps of different nanocrystals as a function of size; the excellent agreement in each case establishes the validity of the present approach over the entire range of nanocrystal sizes. We have also carried out a similar analysis on all the II-VI semiconductors starting with the more accurate FP-LAPW results as inputs compared to the previous approach. ${ }^{9}$ As these results are found to be slightly different and possibly more accurate in comparison to the earlier results, the new parameter values for the II-VI series are also reported here.

\section{METHODOLOGY}

$A b$ initio band structures of all the III-V as well as II-VI compound semiconductors with the zinc blende structure were obtained using the FP-LAPW method. Self-consistency was achieved using $30 \mathrm{k}$ points in the irreducible Brillouin zone. In order to obtain a realistic TB model, we first calculated the band dispersions and density of states (DOS). The atomic orbital contributions to the valence and conduction bands in the band dispersions and the DOS were determined in terms of the orbital-projected band structure, the so-called fatbands, and the partial densities of states (PDOS), respectively. An analysis of these results establishes the minimal orbital basis for the TB model. To obtain a guideline for the range of relevant hopping interactions necessary for reliable descriptions of the valence and conduction bands, we carried out NMTO calculations that provide TB Hamiltonians derived in a first-principles way by constructing atom-centered, short-ranged Wannier orbitals, namely the NMTOs.
The TB Hamiltonian is given by

$$
\mathbf{H}=\sum_{i l} \epsilon_{i l} a_{i l}^{\dagger} a_{i l}+\sum_{i j} \sum_{l l^{\prime}}\left(t_{i j}^{l l^{\prime}} a_{i l}^{\dagger} a_{j l^{\prime}}+\text { H.c. }\right),
$$

where $a_{i l}^{\dagger}$ and $a_{i l}$ are, respectively, the creation and annihilation operators for electrons at the atomic site, $i$ in the $l$ th orbital. The on-site energy for the orbital $l$ at the site $i$ is given by $\epsilon_{i l}$. The hopping interaction strengths $t_{i j}^{l l^{\prime}}$ depend on the type of orbitals and geometry of the lattice and are parametrized using the Slater-Koster parametrization scheme. ${ }^{13}$ We start with the estimates of the values of on-site energies and the hopping integrals obtained from NMTO derived TB Hamiltonian and then carry out a least-squared error minimization fitting procedure at a number of high symmetry points in the band dispersion curves to fit the band dispersion obtained from the FP-LAPW method. The parameters thus obtained are used for calculations of the electronic structure of nanocrystals.

We generated the clusters consisting of a central anion surrounded by the four nearest neighbor cations, followed progressively by alternate shells of anion and cations, similar to our previous studies on the different II-VI semiconductors, ${ }^{11,12}$ and on Mn doped GaAs. ${ }^{25}$ The effective diameter, $d$, of the nanocrystal is calculated assuming that the particles are spherical in shape using the formula

$$
d=a\left[\frac{3 N_{a t}}{4 \pi}\right]^{1 / 3},
$$

where $a$ is the bulk lattice parameters and $N_{a t}$ is the number of atoms in the nanocrystal. The largest nanocrystal for which the DOS was calculated has $N_{a t} \sim 10000$ atoms and $d \sim 7.5 \mathrm{~nm}$ containing approximately 65000 orbitals. As it is virtually impossible to perform a complete diagonalization of such a large matrix, we obtain the eigenvalue spectrum using the Lanczos algorithm. ${ }^{26}$ We passivate the clusters with hydrogen atoms at the outermost layer to remove the dangling bonds and obtain the eigenvalue spectrum for clusters with different sizes. From the eigenspectrum, the top of the valence states (TVS) and the bottom of the conduction states (BCS) are obtained and the bare bandgap is calculated as the difference between them. However, the bare bandgap cannot be directly obtained from experimental data, usually based on optical absorption spectra, due to the presence of the excitonic peak close to the absorption edge. ${ }^{6}$ Hence, we compare the theoretically obtained excitonic peak position with the experimental results. The excitonic peak position is determined theoretically by subtracting the binding energy of the exciton from the calculated bare bandgap. The excitonic binding energy is given by the equation $E_{c}=3.572 e / \varepsilon d$, where $\varepsilon$ is the dielectric constant of the material and $d$ is the diameter of the nanocrystal. ${ }^{27}$

\section{RESULTS AND DISCUSSION}

\section{A. TB parametrization of the bulk electronic structure}

In order to obtain the physical and realistic model and accurate parameter values for various semiconductors, we start with the analysis of bulk band structure of these semi- 

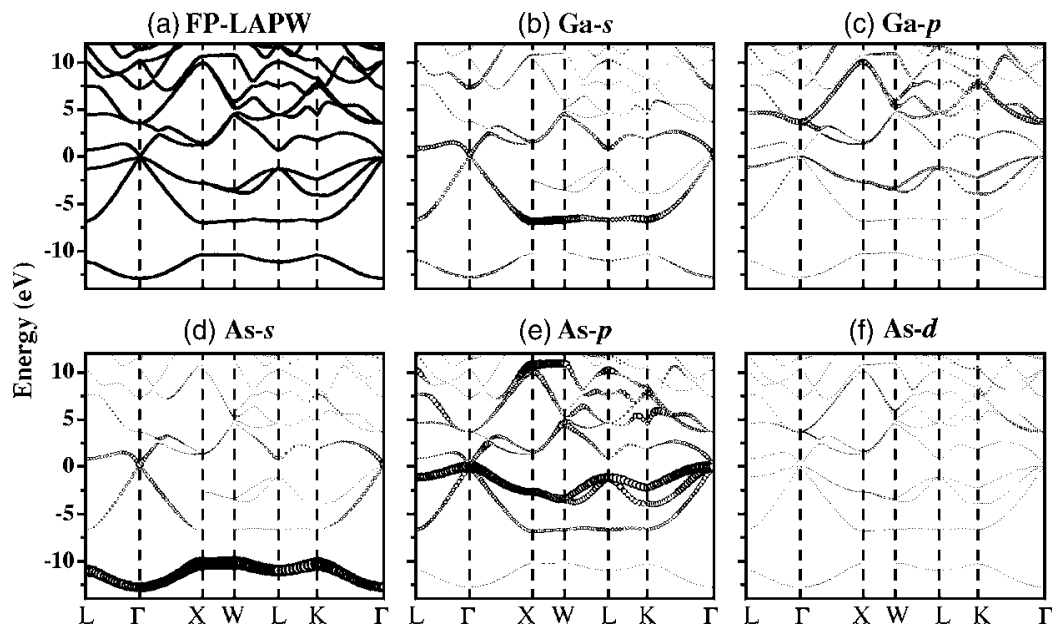

(e) As-p
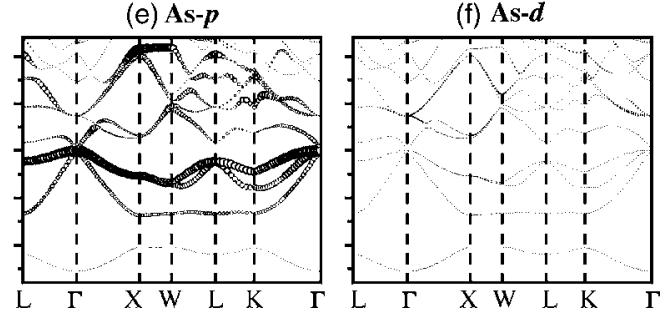

FIG. 1. (a) FP-LAPW band dispersions for the zinc blende structure of GaAs along the various symmetry lines. (b)-(f) Fat bands showing, respectively, the contribution of Ga- $s, \mathrm{Ga}-p$, As- $s$, As- $p$, and As- $d$ on various bands. conductors. Since the successive steps involved in the analyses are similar for each of the compounds, we illustrate the various steps using the example of GaAs. The band dispersions obtained from the FP-LAPW method along various symmetry directions for GaAs are shown in Fig. 1(a) with the zero of the energy scale referring to the top of the valence band. The calculated results show a direct bandgap of about $0.3 \mathrm{eV}$, grossly underestimating the experimental value of $1.4 \mathrm{eV}$. It is well known that LDA methods underestimate the bandgap. However, since we are primarily interested in estimating the change in the bandgap of a nanocrystal compared to that of the bulk, we do not attempt to correct the bandgap artificially, implicitly assuming that the errors in estimating the absolute bandgaps cancel out to a large extent. This assumption turns out to be a reasonable one, as will be shown later in the text, for the present series of III-V compounds and also for the II-VI compounds. ${ }^{11}$

In order to understand the orbital contributions to the bulk band structure, we obtain contribution of each of the orbitals to the band wave functions at each of the energy and momentum points, shown in terms of fat bands, in Figs. 1(b)-1(f). In these panels, though the band dispersions are the same as in Fig. 1(a), only the fatness associated with each band varies, with the size of the circles indicating the amount of the particular orbital character for that band at that $\mathrm{k}$ point. For example, it can be seen that the lowest conduction band between 0 and $3 \mathrm{eV}$ has contributions mainly from $\mathrm{Ga}-s$ (Fig. 1(b)), with substantial mixing from As-s (Fig. 1(d)) and As- $p$ (Fig. 1(e)) states. However, the main part of As- $s$ appears as nearly flat band at an energy of $-11 \mathrm{eV}$ (Fig. 1(d)). From Figs. 1(b) and 1(e), it can be seen that the three strongly dispersive valence bands between $-7 \mathrm{eV}$ and $0 \mathrm{eV}$ are made up of a mixture of mostly As- $p$ and Ga- $s$ orbitals. Though mostly prominent in the highly dispersive bands above the lowest conduction band, Ga- $p$ contributions to some parts of the lowest conduction band (e.g., along $\Gamma-X$ and near the $\mathrm{X}$ point) as well as to the top two valence bands are not negligible (Fig. 1(c)). Hence, it is necessary to include at least the $s p^{3}$ orbitals of both $\mathrm{Ga}$ and As in the basis. Also the contribution of As-d (Fig. 1(f)) to the bands of interest, though not very prominent, cannot be neglected, especially in the conduction band region. Hence, we also included As- $d$ in the basis. The contribution of Ga- $d$ (not shown in the figure), however, is virtually absent in the bands of interest, suggesting Ga $s p^{3}$-As $s p^{3} d^{5}$ as the suitable basis, in contrast to the previously supported ${ }^{7} s p^{3} d^{5} s^{*}$ $s p^{3} d^{5} s^{*}$ basis.

While the atomic orbital contributions to the band wave functions along the high symmetry directions in terms of the fat bands provide a clear suggestion for the suitable basis for the system, this can be further supported by the analysis of the atomic orbital contributions to the overall momentum averaged electronic structure in terms of various PDOS shown in Fig. 2. Panel (a), with Ga-derived PDOS, shows that the bottom of the conduction band as well as the sharp DOS feature at about $-7 \mathrm{eV}$ are dominated by Ga- $s$ states. Ga- $p$ states contribute significantly throughout the conduction and valence states, while Ga- $d$ has very little contribution in these energy ranges. The lower panel (b) clearly shows the dominance of the As- $p$ states in determining the valence band states, while the conduction states have significant contributions from As $s, p$, and $d$ states; in particular, we find that As- $d$ states contribute nearly as much as the As- $s$ states in the conduction band region.

Having determined the relevant basis for the parametrized TB model, we carried out a least-squared-error fit of the FP-LAPW band dispersions in terms of the dispersions of this TB model by systematically varying the electronic parameters (on-site and hopping parameters) of the nearest neighbor (nn) TB Hamiltonian. The fitting was carried out in two steps. First we carried out a fitting of all the 13 bands arising primarily from the Ga-s and $p$ and the As- $s, p$, and $d$, though the As- $d$ states lie high in energy. This inclusion of As- $d$ in the first step ensures that we use a realistic value of As- $d$ for the fitting. In the next step, we fix the As- $d$ on-site energy and reoptimize the parameters to fit the lowest eight bands, in order to provide the most accurate description of the relevant valence and conduction states, primarily arising from the $s$ and $p$ orbitals of Ga and As. The best fit obtained this way is shown in Fig. 3(a). From the figure, it can be seen that though the basic features of the valence and the lowlying conduction bands are captured in this approach, there are too many important and gross discrepancies, such as, the curvature of the lowest conduction band at the $\Gamma$ point, in the TB results compared to the ab initio results. We have highlighted these discrepancies by boxes marked around such discrepancies in the figure. 


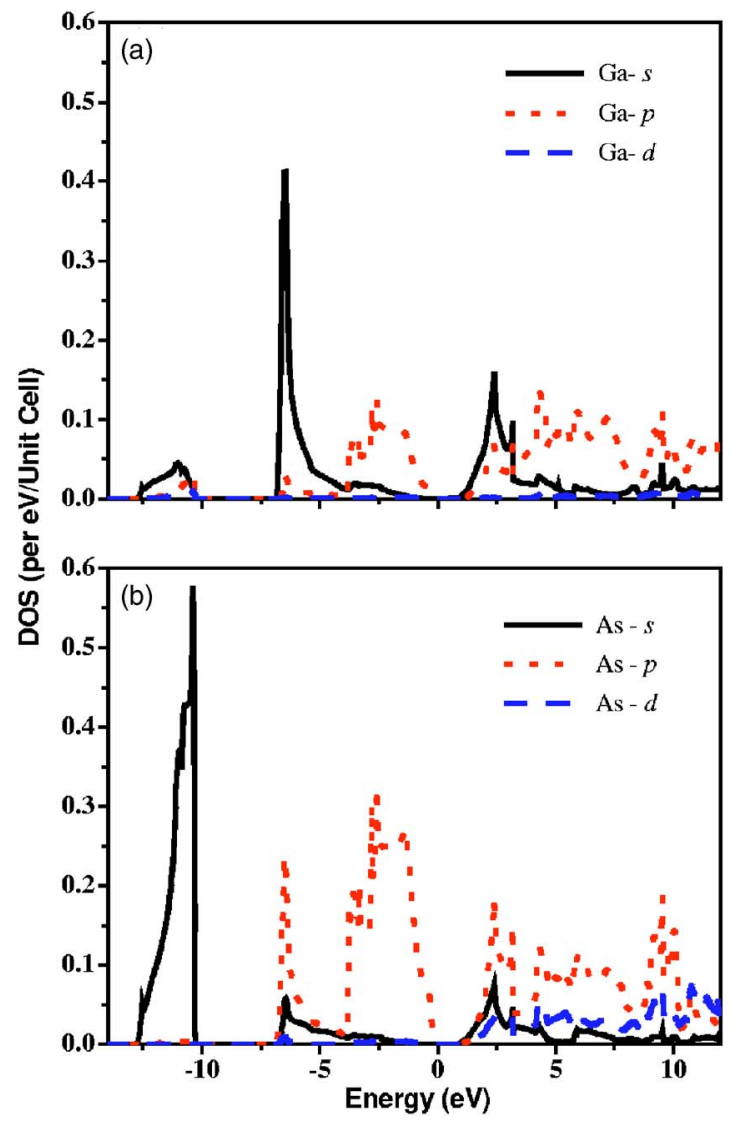

FIG. 2. (Color online) PDOS corresponding to atomic various orbitals in GaAs. In (a), the solid line, dotted line, and the dashed line represents PDOS of Ga- $s, \mathrm{Ga}-p$, and Ga- $d$ orbitals, respectively. In (b), the solid line, dotted line, and dashed line represents the PDOS of As- $s$, As- $p$, and As- $d$, respectively.

These significant discrepancies suggest that the model adopted here misses out on some important interactions, thereby lacking the desired level of accuracy. Such problems have often prompted other groups to increase the basis, for example, by the ad hoc inclusion of an $s^{*}$ orbital on the cationic site. ${ }^{7}$ However, this does not remedy the limitations of the model, as confirmed by us by obtaining the best description TB dispersions with Ga $s p^{3} s^{*}$-As $s p^{3} d^{5}$ basis in comparison to the ab initio approach; this comparison is shown in Fig. 3(b).

In order to obtain an insight into the possible origin of these discrepancies, we carried out analysis based on NMTO calculations that provide us a systematic and ab initio way to construct real space (RS) Hamiltonian by the Fourier transformation from the usual momentum-space Hamiltonian. The real space Hamiltonian, generated in this manner, contains all different interactions, ranging from the nearestneighbor to the farthest interaction. However, it is possible to truncate the RS Hamiltonian at various distances, corresponding to different-sized real-spaced clusters and back Fourier transform the truncated RS Hamiltonian to get the corresponding tight-binding band dispersions. The tight binding bands obtained from such a truncated Hamiltonian, when compared with the band dispersions obtained from the complete calculation provide an understanding of the impor- (a) $\operatorname{Ga}\left(s p^{3}\right)-A s\left(s p^{3} d^{5}\right)$ nn only

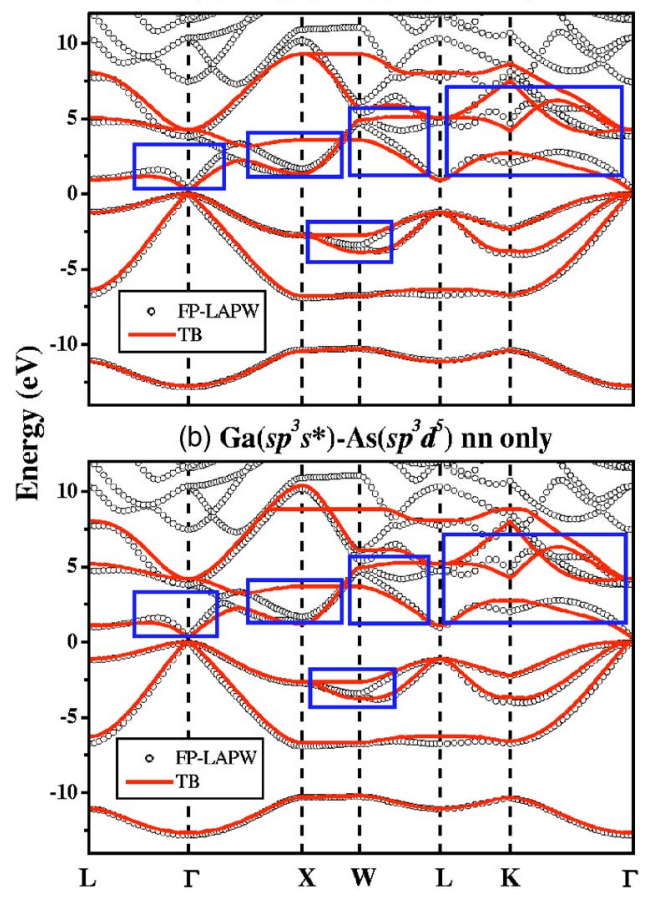

FIG. 3. (Color online) Comparisons of band dispersions obtained for the zinc blende structure of GaAs, from FP-LAPW and from TB fitting for the nearest neighbor interactions only in the (a) $s p^{3}-s p^{3} d^{5}$-orbital basis and (b) $s p^{3} s^{*}-s p^{3} d^{5}$-orbital basis on Ga and As, respectively. The open circles represent the FP-LAPW calculation and the solid line represents the TB calculation.

tant interactions present in a given system. The results of such analysis for GaAs are shown in Fig. 4. From panel (a), it can be observed that the shortest-ranged Hamiltonian, including only the nearest neighbor $\mathrm{Ga}$ - As interactions is not able to describe the conduction band dispersions at all; one can also notice significant mismatches within the valence band region as well. An extension of the range of the Hamiltonian to additionally include As-As next nearest neighbor interactions (panel (b)) improves significantly the description of the conduction band states, thereby establishing the importance of As-As interactions in determining the electronic structure of this compound. However, we still find substantial discrepancies and hence include the $\mathrm{Ga}-\mathrm{Ga}$ interaction. In this case, we find a substantial overall improvement in the descriptions of the valence band dispersions as well as the conduction bands, as shown in panel (c). This suggests that the most reasonable parametrized TB Hamiltonian with the Ga $s p^{3}$-As $s p^{3} d^{5}$ basis should include the nearest neighbor and the second nearest neighbor interactions, where suitably chosen interaction parameters will be able to provide a satisfactory description to the electronic structure of GaAs via renormalization of these parameters to include effects of all those interactions that are neglected in this minimal basis, short-ranged TB model.

The above-mentioned expectation is comprehensively justified by the results shown in Fig. 5, where we present the fits to the $a b$ initio band dispersions within three different parametrized TB models, namely (a) Ga $s p^{3}$-As $s p^{3} d^{5}$ basis with $\mathrm{Ga}-\mathrm{As}$ and $\mathrm{As}-\mathrm{As}$ interactions; (b) Ga $s p^{3} s^{*}$ - 
(a) Ga-As

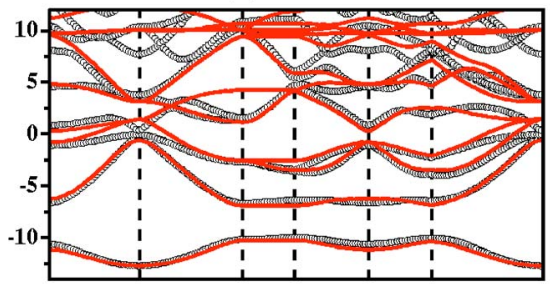

(b) Ga-As + As-As

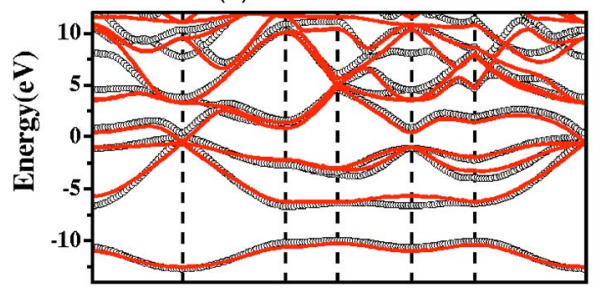

(c) Ga-As + Ga-Ga + As-As

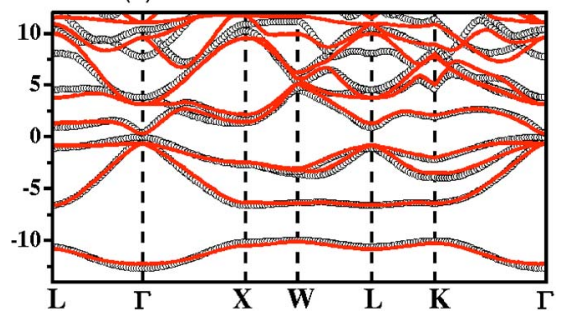

FIG. 4. (Color online) A comparison of TB bands of various hopping ranges computed within the NMTO scheme with the LDA band structure. The LDA band structure is shown as open circles, while the TB bands are shown as a solid line. The hopping ranges include (a) nearest neighbor interaction, (b) nearest neighbor and As-As interaction, (c) nearest neighbor and $\mathrm{Ga}-\mathrm{Ga}$ as well as As-As interactions in the $s p^{3}-s p^{3} d^{5}$-orbital basis on Ga and As, respectively. (a) Ga $\left(s p^{3}\right)-\mathbf{A s}\left(s p^{3} d^{5}\right)+\mathbf{A s}-\mathbf{A s}$

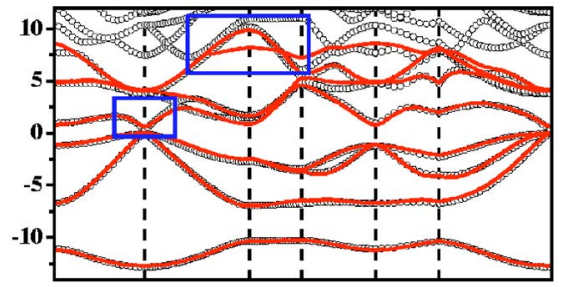

(b) $\mathbf{G a}\left(s p^{3} s^{*}\right)-\mathbf{A s}\left(s p^{3} d^{5}\right)+\mathbf{A s}-\mathbf{A s}$

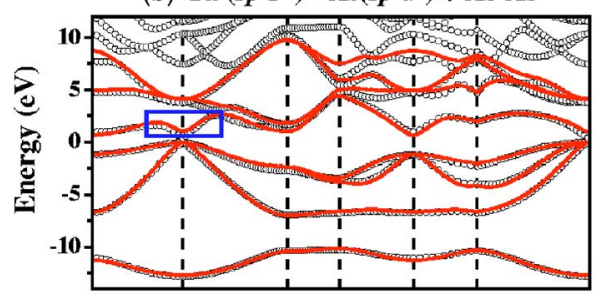

(c) $\mathbf{G a}\left(s p^{3}\right)-\mathbf{A s}\left(s p^{3} d^{5}\right)+\mathrm{As}-\mathrm{As}+\mathrm{Ga}-\mathrm{Ga}$

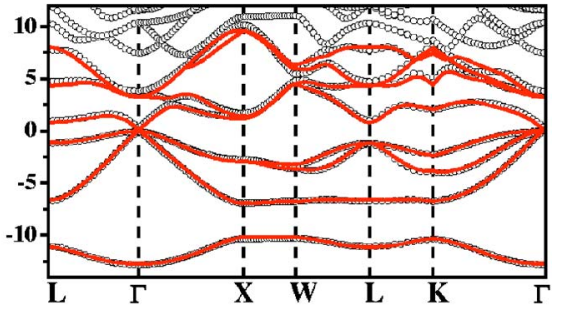

FIG. 5. (Color online) Comparisons of band dispersions obtained from FP-LAPW and TB error minimized fit obtained using nearest neighbor and (a) As-As interactions in the $s p^{3}-s p^{3} d^{5}$-orbital basis, (b) As-As interactions in the $s p^{3} s^{*}-$ $s p^{3} d^{5}$-orbital basis, and (c) $\mathrm{Ga}-\mathrm{Ga}$ as well as As-As interactions in the $s p^{3}-s p^{3} d^{5}$-orbital basis on $\mathrm{Ga}$ and As, respectively. The open circles represent the FP-LAPW calculation and the solid line represents the TB calculation. (a) AIP

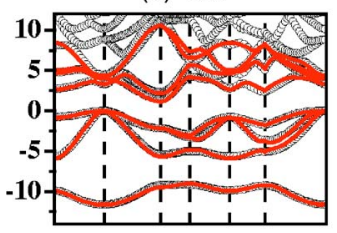

(d) $\mathrm{GaN}$

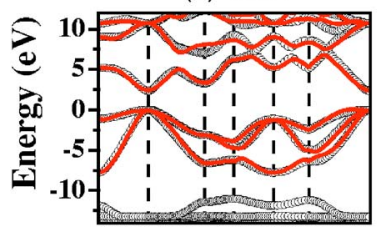

(g) GaSb

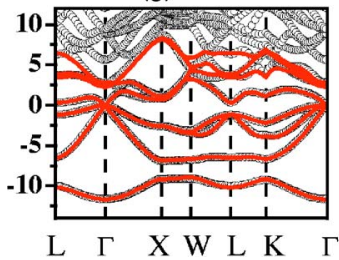

(b) AlAs

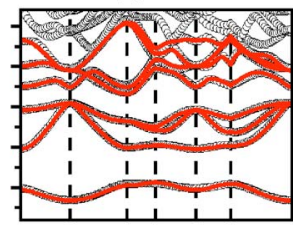

(e) GaP

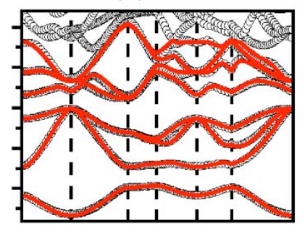

(h) InP

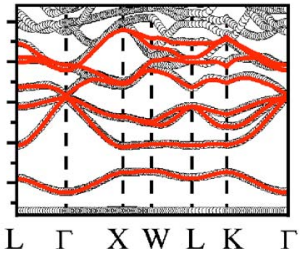

(c) AISb

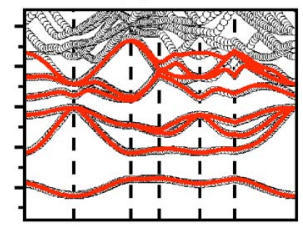

(f) GaAs

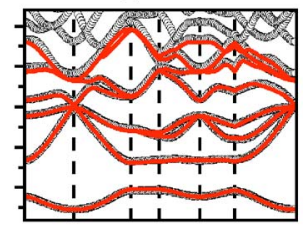

(i) InAs

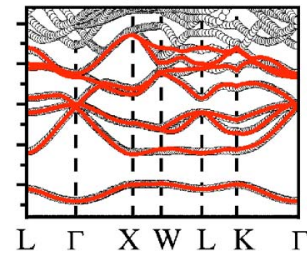

FIG. 6. (Color online) Band dispersions of various III-V semiconductors obtained using FPLAPW (open circles) and TB fit (solid line) obtained using the optimized parameters, given in Table I. 
TABLE I. Parameters (in eV) obtained from TB least-squared-error fitting procedure for the various III-V semiconductors. The parameters are obtained with energy zero at the valence band maximum.

\begin{tabular}{|c|c|c|c|c|c|c|c|c|c|}
\hline & AlP & AlAs & $\mathrm{AlSb}$ & $\mathrm{GaN}$ & $\mathrm{GaP}$ & GaAs & Gasb & InP & InAs \\
\hline$s_{c}$ & 4.89 & 4.22 & 4.37 & 6.02 & 1.73 & 1.12 & 1.01 & 1.50 & 1.04 \\
\hline$p_{c}$ & 8.44 & 8.24 & 5.71 & 10.60 & 7.45 & 7.79 & 6.38 & 7.00 & 5.76 \\
\hline$s_{a}$ & -8.24 & -9.12 & -8.26 & -9.58 & -8.48 & -10.15 & -9.50 & -8.00 & -9.74 \\
\hline$p_{a}$ & -0.62 & -0.14 & 0.69 & -0.51 & -0.04 & -0.31 & -1.04 & 0.53 & 0.06 \\
\hline$d_{a}$ & 9.43 & 9.31 & 6.80 & - & 7.87 & 6.80 & 7.25 & 9.00 & 8.25 \\
\hline$s_{c} s_{a} \sigma$ & -1.39 & -1.10 & -1.26 & -0.53 & -1.78 & -1.26 & -0.47 & -1.04 & -0.82 \\
\hline$s_{c} p_{a} \sigma$ & 2.42 & 2.41 & 2.67 & 1.76 & 2.81 & 2.78 & 2.28 & 2.30 & 2.34 \\
\hline$s_{c} d_{a} \sigma$ & -1.65 & -1.76 & -2.63 & - & -2.27 & -2.00 & -1.93 & -1.72 & -1.81 \\
\hline$p_{c} p_{a} \sigma$ & 2.79 & 2.42 & 3.01 & 3.66 & 3.55 & 2.86 & 2.23 & 2.99 & 2.88 \\
\hline$p_{c} p_{a} \pi$ & -0.57 & -0.79 & -0.70 & -1.12 & -0.83 & -1.04 & -0.74 & -0.53 & -0.63 \\
\hline$p_{c} d_{a} \sigma$ & -0.09 & -1.33 & -1.71 & - & -1.60 & -0.85 & -1.02 & -0.05 & -0.04 \\
\hline$p_{c} d_{a} \pi$ & 2.47 & 1.71 & 1.43 & - & 1.80 & 1.39 & 1.45 & 2.12 & 2.00 \\
\hline$p_{c} s_{a} \sigma$ & -1.87 & -1.47 & -1.21 & -4.50 & -1.81 & -0.48 & -0.22 & -1.63 & -1.19 \\
\hline$s_{c} s_{c} \sigma$ & -0.23 & -0.28 & -0.46 & -0.30 & -0.36 & -0.27 & -0.12 & -0.18 & -0.21 \\
\hline$s_{c} p_{c} \sigma$ & 0.23 & 0.34 & 0.38 & 0.01 & 0.11 & 0.20 & 0.03 & 0.15 & 0.05 \\
\hline$p_{c} p_{c} \sigma$ & 0.35 & 0.57 & 0.35 & 1.39 & 0.48 & 0.13 & 0.32 & 0.00 & 0.05 \\
\hline$p_{c} p_{c} \pi$ & -0.34 & -0.15 & -0.14 & -0.57 & -0.23 & -0.01 & -0.22 & -0.20 & -0.23 \\
\hline$s_{a} s_{a} \sigma$ & -0.08 & -0.08 & -0.01 & -3.24 & 0.00 & -0.01 & -0.15 & -0.12 & -0.10 \\
\hline$s_{a} p_{a} \sigma$ & 0.25 & 0.05 & 0.01 & 1.31 & 0.14 & 0.10 & 0.22 & 0.12 & 0.16 \\
\hline$s_{a} d_{a} \sigma$ & -0.05 & 0.00 & -0.08 & - & -0.07 & -0.16 & -0.39 & -0.16 & -0.11 \\
\hline$p_{a} p_{a} \sigma$ & 0.49 & 0.28 & 0.32 & 1.21 & 0.46 & 0.17 & 0.35 & 0.47 & 0.41 \\
\hline$p_{a} p_{a} \pi$ & -0.04 & -0.04 & -0.01 & -0.02 & -0.02 & -0.03 & -0.01 & -0.00 & -0.01 \\
\hline$p_{a} d_{a} \sigma$ & -0.13 & -0.33 & -0.22 & - & -0.30 & -0.32 & -0.32 & -0.02 & -0.00 \\
\hline$p_{a} d_{a} \pi$ & 0.00 & 0.08 & 0.00 & - & 0.05 & 0.10 & 0.11 & 0.03 & 0.01 \\
\hline$d_{a} d_{a} \sigma$ & -1.16 & -0.76 & -0.85 & - & -1.02 & -0.85 & -0.75 & -1.06 & -0.85 \\
\hline$d_{a} d_{a} \pi$ & 0.33 & 0.50 & 0.37 & - & 0.42 & 0.49 & 0.39 & 0.34 & 0.27 \\
\hline$d_{a} d_{a} \delta$ & -0.01 & -0.01 & -0.01 & - & -0.01 & -0.14 & -0.01 & -0.00 & -0.00 \\
\hline$d_{c}$ & $d_{c} s_{a} \sigma$ & $d_{c} p_{a} \sigma$ & $d_{c} p_{a} \pi$ & $s_{c} d_{c} \sigma$ & $p_{c} d_{c} \sigma$ & $p_{c} d_{c} \pi$ & $d_{c} d_{c} \sigma$ & $d_{c} d_{c} \pi$ & $d_{c} d_{c} \delta$ \\
\hline $\mathrm{GaN} 13.51$ & -1.13 & 2.13 & -1.81 & -0.66 & -1.89 & 0.34 & -2.38 & 0.55 & 0.00 \\
\hline
\end{tabular}

As $s p^{3} d^{5}$ basis with Ga-As and As-As interactions; and (c) $\mathrm{Ga} s p^{3}-\mathrm{As} s p^{3} d^{5}$ basis with $\mathrm{Ga}-\mathrm{As}, \mathrm{Ga}-\mathrm{Ga}$ and $\mathrm{As}-\mathrm{As}$ interactions. Figure 5(c) evidently exhibits the most accurate description of the electronic structure of GaAs over the entire valence and conduction band ranges in terms of the TB model. These results further establish that it is not necessary to introduce the fictitious $s^{*}$ orbital in the basis, as it does not improve anything significantly.

Similar analysis were carried out for all the other III-V systems studied here. In every case, except for GaN we found the model with the cationic $s p^{3}$-anionic $s p^{3} d^{5}$ basis and first and second nearest neighbor interactions to be both necessary and sufficient to provide accurate descriptions of the electronic structures. In the case of GaN, the Ga $s p^{3} d^{5}$ $\mathrm{N} s p^{3}$ basis was found to be the most suitable. The comparison between the $a b$ initio band dispersion and the TB dispersion with the optimized electronic parameter strengths is shown for each of the compounds in Fig. 6, illustrating highly accurate descriptions throughout. The corresponding optimized parameter values are given in Table I.
An earlier study of II-VI compounds employing band dispersions calculated within the linearized muffin-tin orbitals (LMTO) and atomic sphere approximation (LMTO-ASA) as the reference for the electronic structure provided $^{9}$ a TB model based on the $s p^{3} d^{5}$ basis on both cations and anions and the nearest neighbor cation-anion and second nearest neighbor anion-anion interactions only. Noting that FPLAPW provides a more accurate starting point compared to the LMTO-ASA results and that the earlier model for the II-VI compound is slightly different from the present model for the III-V compounds, we have reinvestigated the II-VI series employing FP-LAPW calculations. Carrying out a similarly detailed analysis as presented here for the III-V compounds, we found that the minimal basis for accurate descriptions of electronic structures for the II-VI series consists of the previously employed $s p^{3} d^{5}$ orbitals on both anions and cations and both the second nearest neighbor interactions in addition to the nearest neighbor cation-anion interactions. In essence, the present analyses suggest that TB model of Ref. 9 needs to be extended to include also the 
TABLE II. Parameters (in eV) obtained from TB least-squarederror fitting procedure for the various II-VI semiconductors. The parameters are obtained with energy zero at the valence band maximum.

\begin{tabular}{rrrrrrr}
\hline \hline & $\mathrm{ZnS}$ & $\mathrm{ZnSe}$ & $\mathrm{ZnTe}$ & \multicolumn{1}{c}{$\mathrm{CdS}$} & $\mathrm{CdSe}$ & $\mathrm{CdTe}$ \\
\hline$s_{c}$ & 1.18 & -0.33 & 3.26 & 3.53 & 2.66 & 2.50 \\
$p_{c}$ & 9.79 & 6.60 & 5.05 & 8.46 & 8.28 & 7.16 \\
$d_{c}$ & -6.46 & -6.70 & -6.86 & -7.53 & -7.56 & -7.96 \\
$s_{a}$ & -8.68 & -9.21 & -10.05 & -10.88 & -10.75 & -9.68 \\
$p_{a}$ & 0.47 & 1.35 & -0.22 & -0.41 & -0.49 & -0.62 \\
$d_{a}$ & 11.27 & 10.91 & 9.45 & 13.60 & 10.24 & 9.12 \\
$s_{c} s_{a} \sigma$ & -1.65 & -1.61 & -1.03 & -1.02 & -0.94 & -0.72 \\
$s_{c} p_{a} \sigma$ & 2.41 & 2.41 & 1.36 & 1.83 & 1.90 & 1.77 \\
$s_{c} d_{a} \sigma$ & -1.71 & -1.82 & -1.88 & -2.41 & -1.81 & -1.90 \\
$p_{c} p_{a} \sigma$ & 3.73 & 3.67 & 3.05 & 2.75 & 2.73 & 2.34 \\
$p_{c} p_{a} \pi$ & -0.46 & -0.61 & -0.53 & -0.30 & -0.39 & -0.38 \\
$p_{c} d_{a} \sigma$ & -0.32 & -0.92 & -0.95 & -0.56 & -0.13 & -0.33 \\
$p_{c} d_{a} \pi$ & 3.21 & 2.49 & 1.41 & 2.53 & 2.47 & 2.21 \\
$p_{c} s_{a} \sigma$ & -2.31 & -1.86 & -1.15 & -1.07 & -2.13 & -1.59 \\
$d_{c} s_{a} \sigma$ & -1.42 & -1.49 & -0.52 & -0.68 & -0.65 & -0.52 \\
$d_{c} p_{a} \sigma$ & 0.01 & 0.01 & 0.01 & 0.97 & 0.95 & 0.82 \\
$d_{c} p_{a} \pi$ & -0.13 & -0.10 & -0.06 & -0.40 & -0.34 & -0.25 \\
$s_{c} s_{c} \sigma$ & -0.24 & -0.19 & -0.30 & -0.33 & -0.29 & -0.23 \\
$s_{c} p_{c} \sigma$ & 0.11 & 0.00 & 0.01 & 0.07 & 0.20 & 0.16 \\
$p_{c} p_{c} \sigma$ & 0.01 & 0.01 & 0.29 & 0.31 & 0.34 & 0.14 \\
$p_{c} p_{c} \pi$ & -0.24 & -0.01 & -0.24 & -0.20 & -0.22 & -0.15 \\
$s_{a} s_{a} \sigma$ & -0.05 & -0.02 & 0.04 & 0.00 & -0.07 & -0.07 \\
$s_{a} p_{a} \sigma$ & 0.19 & 0.19 & 0.01 & 0.13 & 0.01 & 0.01 \\
$p_{a} p_{a} \sigma$ & 0.55 & 0.53 & 0.78 & 0.33 & 0.32 & 0.35 \\
$p_{a} p_{a} \pi$ & -0.01 & -0.09 & -0.10 & 0.00 & -0.02 & -0.02 \\
\hline \hline & & & & & & \\
\hline
\end{tabular}

second nearest neighbor cation-cation interaction in order to provide a comparable level of accuracy in describing the FP-LAPW results. While we do not present the details of the analysis for the II-VI series here, it being along the same line as presented for the III-V series, we have tabulated the optimized electronic parameter strengths of the TB model in Table II.

\section{B. Electronic structure of nanocrystals}

The bandgap of a finite sized crystal is known to have a pronounced dependence on the size of the crystal in the nanometric regime. This has opened up immense technological possibilities, based primarily on the tunability of the bandgap in the quantum confinement regime. If the electronic parameter strengths as well as the TB model itself remain valid down to such small sizes, the present analysis and results provide a reliable way to understand or even predict such bandgap variations with the size by performing real space calculations for the finite-sized crystals with the same model and parameter values. Encouraged by the previous success in similar studies, ${ }^{9-12,25}$ we have carried out electronic structure calculations for finite sized III-V systems using the Lanczos algorithm as described in Sec. II. Various panels in Fig. 7 show the calculated shifts (open circles) in the bandgap of the nanocrystal relative to the bulk bandgap as a function of size. In order to provide an analytical description of the systematic variation of $\Delta E_{g}$, the calculated results (open circles) were fitted with an empirical expression of the form $1 /\left(a d^{2}+b d+c\right)$, where $a, b$, and $c$ are obtained by fitting. The choice of the expression here, though entirely empirical, was prompted by the $1 / d^{2}$ dependence found in EMA. However, this simple dependence was found to be insufficient to describe the results; therefore we use the simplest extension of the EMA expectation that is able to fit the results accu- (a) AIP

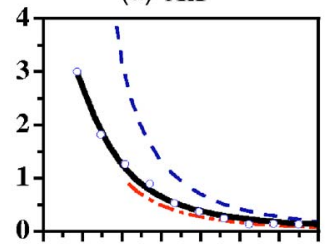

(d) $\mathrm{GaN}$

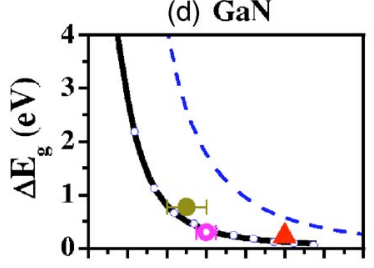

(g) GaSb

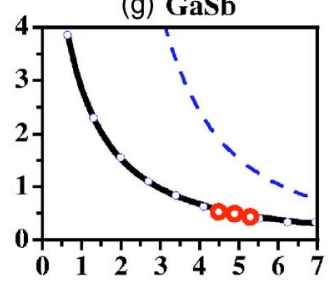

(b) AlAs

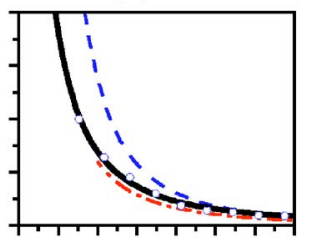

(e) $\mathrm{GaP}$

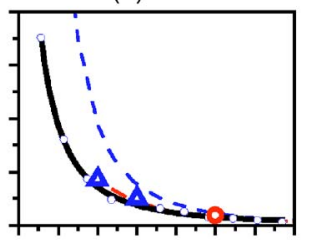

(h) InP

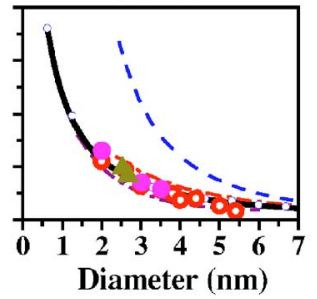

(c) AISb

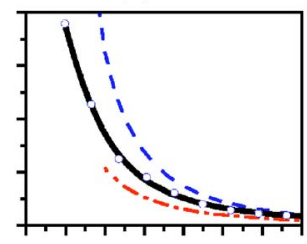

(f) GaAs

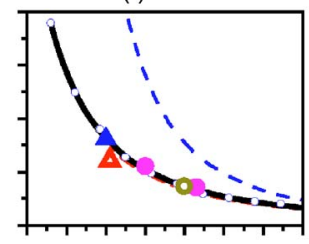

(i) InAs

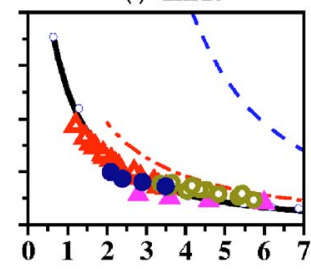

FIG. 7. (Color online) Variation of bandgap of the different III-V nanocrystals obtained from the TB approximation and comparison with experimentally obtained data (panel (d), Refs. 30 (closed circles), 31 (open circles), 32 (open triangle), panel (e) Refs. 17 (open circle), 29 (open triangle), panel (f) Refs. 33 (open circles), 34 (open triangles), 35 (closed circles), 36 (closed triangles), panel (g) Ref. 37 (open circles), panel (h) Refs. 38 (open circles), 39 (closed circles), 40 (closed triangles), panel (i) Refs. 18 (open circles), 41 (open triangles), 42 (closed circles), 22 (closed triangles)). The curves obtained from EMA are shown by the dashed line and the $s p^{3} d^{5} s^{*}$ calculation are shown by dashed dotted lines. 
TABLE III. Parameters obtained from fitting the variation of bandgap for the different III-V semiconductors using the form $\Delta E_{g}=1 /\left(a d^{2}+b d+c\right)$.

\begin{tabular}{lccc}
\hline \hline & $a\left(\mathrm{~nm}^{-2} \mathrm{eV}^{-1}\right)$ & $b\left(\mathrm{~nm}^{-1} \mathrm{eV}^{-1}\right)$ & $c\left(\mathrm{eV}^{-1}\right)$ \\
\hline $\mathrm{AlP}$ & 0.1605 & -0.0588 & 0.2663 \\
$\mathrm{AlAs}$ & 0.0997 & 0.1477 & 0.0279 \\
$\mathrm{AlSb}$ & 0.1258 & -0.0649 & 0.2072 \\
$\mathrm{GaN}$ & 0.3716 & -0.2336 & 0.2172 \\
$\mathrm{GaP}$ & 0.1969 & 0.2631 & 0.0728 \\
$\mathrm{GaAs}$ & 0.0359 & 0.1569 & 0.1564 \\
$\mathrm{GaSb}$ & 0.0357 & 0.1963 & 0.1175 \\
$\mathrm{InP}$ & 0.0461 & 0.3153 & 0.0623 \\
$\mathrm{InAs}$ & 0.0374 & 0.2569 & 0.1009 \\
\hline \hline
\end{tabular}

rately enough. The values of $a, b$, and $c$ for different semiconductors are shown in Table III; these values allow one to calculate the change in bandgap of a nanocrystal with any specific size. The curve obtained by fitting is shown in the various panels of Fig. 7 as a solid thick line. In this figure, we also compare this calculated curve with experimental data, wherever available, different symbols representing data from different publications. We have also compiled in these figures calculated results from other approaches, such as those based on EMA (dashed lines in each panel), TB model using a $s p^{3} d^{5} s^{*}$ basis $^{8}$ (dotted lines) and semiempirical pseudopotential method ${ }^{5}$ available only for InP (dashed dotted line). These comparisons clearly show that the present TB model provides a description of the experimentally observed variation of bandgaps more accurately than the other theoretical approaches. For example, EMA is found to grossly overestimate the bandgaps in every case. The $s p^{3} d^{5} s^{*}$ model is also found to overestimate the bandgap variation compared to the experimental data for InAs (see panel (i) of Fig. 7); in contrast, results from the present model is found to be in striking agreement with the experimental results. For InP, the only other case where extensive experimental results exist, we again find a remarkable agreement with calculated results based on the present model over the entire range of sizes. This establishes the effectiveness of the TB model developed here and reliability of the estimated parameter strengths, even for the study of finite-sized nanocrystals.

As we have a new set of parameter values (Table II) with a slightly different TB model for the II-VI series compared to the earlier report, ${ }^{9}$ we have carried the electronic structure calculations for the nanocrystals of all these II-VI compounds also, for the sake of completion. We find that the new results are in good agreement with the experimental data reported in Ref. 11, earlier. The variation of bandgap in the II-VI semiconductors are also fitted using the same expression $\Delta E_{g}=1 /\left(a d^{2}+b d+c\right)$ and the values of the fitting parameters are shown in Table IV.

In view of the recent experiments on II-VI semiconductors using high-energy spectroscopies, mapping out separately the valence and conduction bands, ${ }^{28}$ it is important to understand the variations of TVS and BCS separately, in addition to probing the changes in the bandgap with size.
TABLE IV. Parameters obtained from fitting the variation of the bandgap for the different II-VI semiconductors using the form $\Delta E_{g}=1 /\left(a d^{2}+b d+c\right)$.

\begin{tabular}{cccc}
\hline \hline & $a\left(\mathrm{~nm}^{-2} \mathrm{eV}^{-1}\right)$ & $b\left(\mathrm{~nm}^{-1} \mathrm{eV}^{-1}\right)$ & $c\left(\mathrm{eV}^{-1}\right)$ \\
\hline $\mathrm{ZnS}$ & 0.2349 & -0.0418 & 0.2562 \\
$\mathrm{ZnSe}$ & 0.0845 & 0.1534 & 0.2128 \\
$\mathrm{ZnTe}$ & 0.0092 & 0.1872 & 0.2396 \\
$\mathrm{CdS}$ & 0.1278 & 0.1018 & 0.1821 \\
$\mathrm{CdSe}$ & 0.0397 & 0.1723 & 0.1111 \\
$\mathrm{CdTe}$ & 0.0275 & 0.2403 & 0.1469 \\
\hline \hline
\end{tabular}

Since the calculated bandgap is constructed from the difference in TVS and BCS, it is straightforward to calculate the variation of the TVS and BCS from our calculations. The variations of TVS (open circles) and BCS (closed circles) as a function of size for the various III-V semiconductors are shown in different panels of Fig. 8. Since we show the change in these quantities as a function of the size with respect to those for the bulk, the zero of the energy axis corresponds to the bulk values; it can be seen from the figures that both TVS and BCS smoothly approach the bulk values with increasing size of the nanocrystals. We also observe that the shift in BCS is larger than that of the TVS in the largersize regime for most of the systems; this indicates that the shift in the bandgap is dominated by the shift in the conduction band edge in such cases. The predominance of the BCS in determining the variation of the bandgap is easy to understand in terms of effective masses of electrons and holes. First, we note that the energy variation of electron or hole states are related inversely to the corresponding effective masses; in other words, the shifts in the valence and conduction states are controlled by $1 / m_{h}^{*}$ and $1 / m_{\rho}^{*}$, respectively. Since the $m_{e}^{*}$ is significantly larger than $m_{h}^{*}$ for the III-V compounds, the conduction band is affected more pronouncedly compared to the valence band with a change in the size. This argument is valid only for the larger-size limit where effective mass, determined for the bulk material remains to be a relevant quantity, even for the nanocrystals. However, in the smaller size regime the trend appears to be reversed in several cases, such as in GaAs (Fig. 8(f)), with the shift in the BCS being less than that in the TVS. This change in the behavior with size can be understood in the following way. At large sizes, the BCS is defined by the states belonging to the $\Gamma$ point of the bulk band structure (see Fig. 6(f) for GaAs), which has a low effective electron mass, being dominantly contributed by the Ga-s states. However, a rapid upward movement of these states with decreasing size inevitably makes BCS to be contributed primarily by the states belonging to $\mathrm{X}$ point of the bulk band structure (see Fig. 6(f)) which evidently corresponds to a larger effective mass, being primarily contributed by the Ga- $p$ states and having a relatively flat dispersion. Therefore, this leads to a relatively less pronounced change in the BCS in the smaller size regime.

\section{CONCLUSIONS}

In this work, we present a systematic development of parametrized tight-binding model for an accurate description of 
(a) AIP

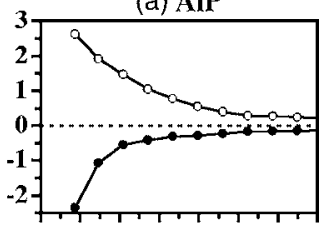

(d) $\mathrm{GaN}$

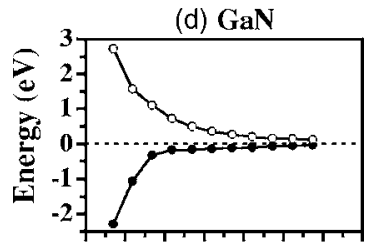

(g) GaSb

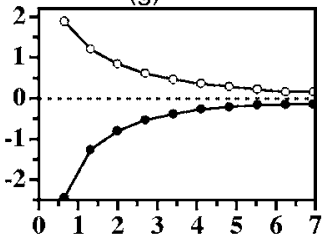

(b) AlAs

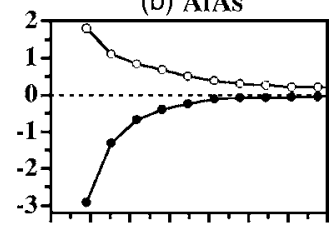

(e) $\mathbf{G a P}$

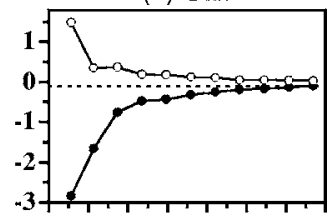

(h) InP

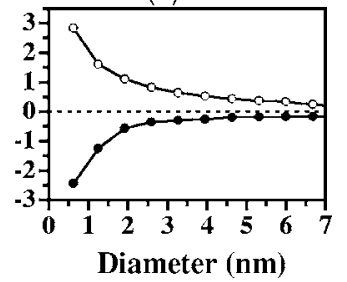

(c) AISb

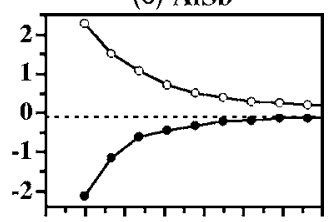

(f) $\mathrm{GaAs}$

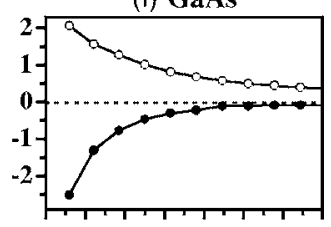

(i) InAs

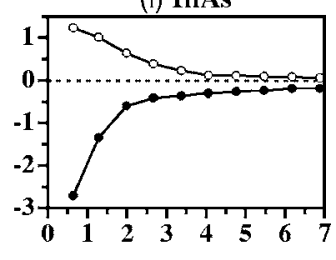

FIG. 8. Difference in TVS (open circles) and BCS (closed circles) from the bulk value plotted as a function of the nanocrystal size for the various III-V semiconductors. The solid line is a guide to eye connecting the data smoothly. the electronic structure of group III-V semiconductors. We analyze the nature and origin of bonding as well as the atomic orbital contributions to each band eigenstates to arrive at the necessary minimal model involving $s p^{3}$ orbitals at the cationic sites and $s p^{3} d^{5}$ orbitals at the anionic sites, obviating the use of any fictitious $s^{*}$ orbital in the basis. We find that though the nearest-neighbor-only model provides an approximate description of the $a b$ initio band dispersions over a wide energy range, it is necessary to include both the cationcation and anion-anion second nearest neighbor interactions to obtain a satisfactorily accurate description of $a b$ initio band dispersions. We have also performed a similar analysis for the II-VI semiconductors using the more accurate FPLAPW $a b$ initio band structure in contrast to the previously used LMTO method. Using these optimized parameters, we perform real space calculations with the same tight binding model to obtain the variation of the bandgap as a function of the nanocrystal size. A comparison with the available experimental data of the bandgap variation with the size of these nanocrystals exhibits good agreement over the entire range of sizes; in sharp contrast to the results obtained with the
EMA. We have also compared the present results with other calculations and we find that the present results give a better description, wherever these differ. A similar analysis was also carried out on the II-VI semiconductors using the newly obtained parameters and the calculated bandgap variation is are found to match well with existing experimental values. Ideally one would like to extend a similar parametrized tightbinding Hamiltonian approach, not only for an accurate description of the electronic structure of such systems, but also to describe the cohesive energy and geometry optimization; this will, however, require an accurate description of the ionic contributions of the total energy along with the electronic contributions that has been modeled here.

\section{ACKNOWLEDGMENTS}

This work is supported by the Department of Science and Technology, Government of India. We thank P. Blaha, K. Schwarz, P. Dufek, and R. Augustyn for providing the LAPW code. We thank Dr. Aparna Chakrabarti for helpful discussions.
*Also at Jawaharlal Nehru Centre for Advanced Scientific Research, Bangalore-560054, India and Centre for Condensed Matter Theory, Indian Institute of Science; electronic address: sarma@sscu.iisc.ernet.in

${ }^{1}$ (a) Thin Film Solar Cells, edited by K. L. Chopra and S. R. Das (Plenum, New York, 1983; (b) S. Hingorani, V. Pillai, P. Kumar, M. S. Multani, and D. O. Shah, Mater. Res. Soc. Symp. Proc. 28, 1303 (1993).

2 (a) A. L. Efros and A. L. Efros, Sov. Phys. Semicond. 16, 772 (1982); (b) L. E. Brus, J. Chem. Phys. 79, 5566 (1983).

${ }^{3}$ F. Buda, J. Kohanoff, and M. Parrinello, Phys. Rev. Lett. 69, 1272 (1992).
${ }^{4}$ M. V. RamaKrishna and R. A. Friesner, Phys. Rev. Lett. 67, 629 (1991).

5 (a) H. Fu and A. Zunger, Phys. Rev. B 55, 1642 (1997); (b) H. Fu and A. Zunger, Phys. Rev. B 56, 1496 (1997).

${ }^{6}$ P. E. Lippens and M. Lannoo, Phys. Rev. B 39, 10935 (1989).

${ }^{7}$ J. M. Jancu, R. Scholz, F. Beltram, and F. Bassani, Phys. Rev. B 57, 6493 (1998).

${ }^{8}$ G. Allan, Y. M. Niquet, and C. Delerue, Appl. Phys. Lett. 77, 639 (2000).

${ }^{9}$ S. Sapra, N. Shanthi, and D. D. Sarma, Phys. Rev. B 66, 205202 (2002).

${ }^{10}$ S. Sapra, R. Viswanatha, and D. D. Sarma, J. Phys. D 36, 1595 
(2003).

${ }^{11}$ S. Sapra and D. D. Sarma, Phys. Rev. B 69, 125304 (2004).

${ }^{12}$ R. Viswanatha, S. Sapra, B. Satpati, P. V. Satyam, B. N. Dev, and D. D. Sarma, J. Mater. Chem. 14, 661 (2004).

${ }^{13}$ J. C. Slater and G. F. Koster, Phys. Rev. 94, 1498 (1954).

${ }^{14}$ P. Vogl, H. P. Hjalmarson, and J. D. Dow, J. Phys. Chem. Solids 44, 365 (1983).

${ }^{15}$ O. K. Andersen and T. Saha-Dasgupta, Phys. Rev. B 62, R16219 (2000), and references therein.

${ }^{16}$ M. Green and P. O’Brien, J. Mater. Chem. 14, 629 (2004).

${ }^{17}$ S. Gao, J. Lu, N. Chen, Y. Zhao, and Y. Zie, Chem. Commun. (Cambridge) 3064 (2002).

${ }^{18}$ A. A. Guzelian, U. Banin, A. V. Kadavanich, X. Peng, and A. P. Alivisatos, Appl. Phys. Lett. 69, 1432 (1996).

${ }^{19}$ M. A. Malik, P. O’Brien, S. Norager, and J. Smith, J. Mater. Chem. 13, 2591 (2003).

${ }^{20}$ A. Manz, A. Birkner, M. Kolbe, and R. A. Fischer, Adv. Mater.. 12, 569 (2000).

21 (a) A. Bar-Lev, Semiconductors and Electronic Devices, 2nd ed. (Prentice-Hall, New York, 1984). (b) A. Katz, Indium Phosphide and Related Materials: Processing, Technology and Devices (Artech House Publishers, Fitchburg, 1992).

${ }^{22}$ M. Bruchez, Jr., M. Moronne, P. Gin, S. Weiss, and A. P. Alivisatos, Science 281, 2013 (1998).

${ }^{23}$ P. Blaha, K. Schwarz, P. Dufek, and R. Augustyn, wIEn95, Technical University of Vienna, 1995 [improved and updated Unix version of the original copyrighted WIEN code; P. Blaha, K. Schwarz, P. Sorantin, and S. B. Trickey, Comput. Phys. Commun. 59, 399 (1990)].

24 (a) R. Valenti, T. Saha-Dasgupta, J. V. Alvarez, K. Pozgajcic, and C. Gros, Phys. Rev. Lett. 86, 5381 (2001); (b) E. Pavarini, I. Dasgupta, T. Saha-Dasgupta, O. Jepsen, and O. K. Andersen, ibid. 87, 047003 (2001); (c) T. Saha-Dasgupta and R. Valenti, Europhys. Lett. 60, 309 (2002).

${ }^{25}$ S. Sapra, D. D. Sarma, S. Sanvito, and N. A. Hill, Nano Lett. 2, 605 (2002).

${ }^{26}$ J. Cullum and R. A. Willoughby, Lanczos Algorithms For Large
Symmetric Eigenvalue Computations: Vols. I and II (Birkhaüser, Basel, 1985).

${ }^{27}$ L. E. Brus, J. Phys. Chem. 90, 2555 (1986).

28 (a) V. L. Colvin, A. P. Alivisatos, and J. G. Tobin, Phys. Rev. Lett. 66, 2786 (1991); (b) T. van Buuren, L. N. Dinh, L. L. Chase, W. J. Siekhaus, and L. J. Terminello, ibid. 80, 3803 (1998); (c) J. Lüning, J. Rockenberger, S. Eisebitt, J. E. Rubensson, A. Karl, A. Kornowski, H. Weller, and W. Eberhardt, Solid State Commun. 112, 5 (1999); (d) J. Nanda, Ph.D. thesis, Indian Institute of Science, Bangalore, 2000.

${ }^{29}$ O. I. Mićić and A. J. Nozik, J. Lumin. 70, 95 (1996).

${ }^{30}$ L. Grocholl, J. Wang, and E. G. Gillan, Chem. Mater. 13, 4290 (2001).

${ }^{31}$ O. I. Mićić, S. P. Ehrenkiel, D. Bertram, and A. J. Nozik, Appl. Phys. Lett. 75, 478 (1999).

${ }^{32}$ Y. G. Cao, X. L. Chen, J. Y. Li, Y. C. Lan, and J. K. Liang, Appl. Phys. A 71, 229 (2000).

${ }^{33}$ M. A. Olshavsky, A. N. Goldstein, and A. P. Alivisatos, J. Am. Chem. Soc. 112, 9438 (1990).

${ }^{34}$ H. Uchida, C. J. Curtis, and A. J. Nozik, J. Phys. Chem. 95, 5382 (1991).

${ }^{35}$ M. A. Malik, P. O' Brien, S. Norager, and J. Smith, J. Mater. Chem. 13, 2591 (2003).

${ }^{36}$ S. S. Kher and R. L. Wells, Nanostruct. Mater. 7, 591 (1996).

${ }^{37}$ F. M. Lui, T. M. Wang, L. D. Zhang, G. H. Li, and H. X. Han, J. Lumin. 99, 273 (2002).

${ }^{38}$ O. I. Mićić, H. M. Cheong, H. Fu, A. Zunger, J. R. Sprague, A. Mascarenhas, and A. J. Nozik, J. Phys. Chem. B 101, 4904 (1997).

${ }^{39}$ O. I. Mićić, J. R. Sprague, Z. Lu, and A. J. Nozik, Appl. Phys. Lett. 68, 3150 (1996).

${ }^{40}$ O. I. Mićić, K. M. Jones, A. Cahill, and A. J. Nozik, J. Phys. Chem. B 102, 9791 (1998).

${ }^{41}$ U. Banin, Y. W. Cao, D. Katz, and O. Miloo, Nature (London) 400, 542 (1999).

${ }^{42}$ D. V. Talapin, S. K. Poznyak, N. P. Gaponik, A. L. Rogach, and A. Eychmüller, Physica E (Amsterdam) 14, 2337 (2002). 